\title{
Efeito da Administração do Atracúrio sobre a Recuperação do Bloqueio Neuromuscular Induzido pelo Pancurônio *
}

\section{Effect of Atracurium on Pancuronium-Induced Neuromuscular Block Recovery}

\author{
Luís Fernando Rodrigues Maria ${ }^{1}$; Maria Angela Tardelli, TSA ${ }^{2}$; Rita de Cássia Rodrigues, TSA $^{2}$
}

\section{RESUMO}

Maria LFR, Tardelli MA, Rodrigues RC - Efeito da Administração do Atracúrio sobre a Recuperação do Bloqueio Neuromuscular Induzido pelo Pancurônio

JUSTIFICATIVA E OBJETIVOS: Freqüentemente em cirurgias abdominais, na fase de fechamento da parede, há necessidade de doses adicionais de bloqueador neuromuscular. O objetivo deste estudo foi analisar, na vigência de recuperação parcial do bloqueio neuromuscular induzido pelo pancurônio, o efeito da administração de dose complementar de atracúrio sobre a recuperação espontânea do bloqueio neuromuscular.

MÉTODO: Foram estudados 30 pacientes, divididos em dois grupos, 14 pacientes formaram o grupo pancurônio e 16 pacientes, o grupo atracúrio. A função neuromuscular foi monitorizada de forma contínua por acelerometria do músculo adutor do polegar, utilizando a seqüência de quatro estímulos (SQE), através da estimulação supramáxima do nervo ulnar. $A$ indução da anestesia foi feita com propofol, fentanil, pancurônio $0,08 \mathrm{mg}^{\mathrm{kg}} \mathrm{kg}^{-1}$ e a manutenção com $\mathrm{N}_{2} \mathrm{O} 60 \%$ em oxigênio e isoflurano na concentração expirada de $0,5 \%$. Quando a primeira contração da seqüência de quatro estímulos $\left(T_{1}\right)$ recuperou $25 \%$, o grupo pancurônio recebeu pancurônio 0,025 mg. $\mathrm{kg}^{-1}$ e o grupo atracúrio, 0,20 mg. $\mathrm{kg}^{-1}$ de atracúrio. Após a dose complementar foram anotados os tempos para recuperação espontânea de $T_{1}$ igual a 10\%, 25\%, $75 \%$, do índice de recuperação (IR25-75\%) e da relação $T_{4} / T_{1}$ igual a 0,8 .

RESULTADOS: Os tempos de recuperação espontânea após dose complementar de pancurônio ou atracúrio não diferiram quando avaliados pela recuperação de $T_{1}$ em $10 \%$ (45,00 \pm $15,50$ vs $49,69 \pm 9,41), 25 \%(61,64 \pm 18,58$ vs $64,25 \pm 12,51) e$ $75 \%(94,00 \pm 28,52$ vs $84,69 \pm 16,50)$. O IR $25-75 \%(32,36 \pm$ $13,76$ vs $20,44 \pm 9,24)$ e o tempo de recuperação da relação $T_{4} / T_{1}=0,8(176,86 \pm 29,57$ vs 141,50 $\pm 29,57)$ foram menores no grupo do atracúrio.

CONCLUSÕES: Nas condições deste estudo, a complementação com atracúrio não promoveu alteração na recuperação espontânea inicial do bloqueio neuromuscular induzido pelo pancurônio e promoveu diminuição de $20 \%$ no tempo de recuperação total.

* Recebido do (Received from) Hospital São Paulo, SP

1. Mestre em Anestesiologia pela UNIFESP-EPM

2. Professora Adjunta da Disciplina de Anestesiologia, Dor e Terapia Intensiva - UNIFESP EPM

Apresentado (Submitted) em 28 de maio de 2003

Aceito (Accepted) para publicação em 20 de agosto de 2003

Endereço para correspondência (Correspondence to)

Dr. Luís Fernando Rodrigues Maria

Rua Floreal, 395 Agudo Romão

15802-145 Catanduva, SP

(c) Sociedade Brasileira de Anestesiologia, 2004
Unitermos: BLOQUEADORES NEUROMUSCULARES, Adespolarizante: atracúrio, pancurônio; MONITORIZAÇÃO: função neuromuscular

\section{SUMMARY}

Maria LFR, Tardelli MA, Rodrigues RC - Effect of Atracurium on Pancuronium-Induced Neuromuscular Block Recovery

BACKGROUND AND OBJECTIVES: Additional neuromuscular blocker doses are in general needed during wall closing after abdominal surgeries. This study aimed at determining during partial pancuronium-induced neuromuscular block recovery, the effect of additional atracurium dose on spontaneous neuromuscular block recovery.

METHODS: Participated in this study 30 patients divided in two groups: pancuronium group $(n=14)$ and atracurium group $(n=$ 16). Neuromuscular function was continuously monitored by accelerometry of abductor pollicis muscle using TOF to supramaximally stimulate ulnarnerve. Anesthesia was induced with propofol, fentanyl and $0.08 \mathrm{mg} \cdot \mathrm{kg}^{-1}$ pancuronium, and was maintained with $60 \% \mathrm{~N}_{2} \mathrm{O}$ in oxygen and $0.5 \%$ isoflurane expired concentration. When $T_{1}$ returned to $25 \%$ of control, 0.025 $\mathrm{mg} . \mathrm{kg}^{-1}$ pancuronium or $0.20 \mathrm{mg} . \mathrm{kg}^{-1}$ atracurium were administered to pancuronium or atracurium group, respectively. Time for spontaneous $T_{1}$ recovery $=10 \%, 25 \%, 75 \%$ of recovery in$\operatorname{dex}(R / 25-75 \%)$ and time to $T_{4} / T_{1}$ equal 0.8 after complementary dose, were recorded.

RESULTS: There were no differences between groups on $T_{1}$ spontaneous recovery to $10 \%(45.00 \pm 15.50$ vs. $49.69 \pm 9.41)$, $25 \%(61.64 \pm 18.58$ vs. $64.25 \pm 12.51)$ and $75 \%(94.00 \pm 28.52$ vs. $84.69 \pm 16.50)$. Recovery index (RI $25-75 \%)$ and time to $T_{4} / T_{1}=0.8$ were shorter in the atracurium group.

CONCLUSIONS: In this study, atracurium complementation has made no difference in initial spontaneous recovery of pancuronium-induced neuromuscular block, but has decreased total recovery time in $20 \%$.

Key Words: MONITORING: neuromuscular function; NEUROMUSCULAR BLOCKERS, Non-depolarizing: atracurium, pancuronium

\section{INTRODUÇÃO}

A utilização dos bloqueadores neuromusculares em anestesia geral tem como benefício promover o relaxamento necessário à intubação traqueal, assim como, facilitar a abordagem do campo operatório, sem que seja necessário um plano anestésico profundo ${ }^{1}$.

O pancurônio é um bloqueador neuromuscular adespolarizante da classe química esteróide e se caracteriza por promover um bloqueio neuromuscular de longa duração, sendo ideal para procedimentos com previsão de duração superior a quatro horas ${ }^{2,3}$. 
O atracúrio pertence à classe química dos benzilisoquinolínicos e possui duração clínica menor que a do pancurônio, apresentando como vantagem adicional pouca dependência de órgãos para sua eliminação ${ }^{4}$.

Ao término de um procedimento intra-abdominal, o grau de relaxamento da musculatura da área cirúrgica é muito importante para o adequado fechamento da cavidade. Freqüentemente, nesta fase da anestesia, há necessidade de doses adicionais de bloqueador neuromuscular com o objetivo de intensificar o relaxamento muscular ${ }^{5}$. Este fato adquire particular importância quando a complementação do bloqueio neuromuscular é realizada com agentes de duração prolongada. A repetição de um bloqueador neuromuscular adespolarizante como o pancurônio poderia, por sua longa duração, implicar tempo prolongado para o completo retorno da força motora e restabelecimento da função ventilatória normal. Nesse momento, a utilização de atracúrio, ao invés do pancurônio, para a complementação de bloqueio neuromuscular induzido pelo pancurônio poderia, teoricamente, resultar em recuperação mais rápida da função motora ${ }^{6,7}$.

O objetivo deste estudo foi analisar, na vigência de recuperação parcial do bloqueio neuromuscular induzido pelo pancurônio, o efeito da administração de dose complementar de atracúrio sobre a recuperação espontânea do bloqueio neuromuscular, em comparação ao efeito de dose complementar de pancurônio.

\section{MÉTODO}

Após aprovação pela Comissão de Ética, foram estudados 30 pacientes, de ambos os sexos, com idades entre 18 e 65 anos, peso com variação até $30 \%$ do peso ideal, estado físico ASA I ou II, com dosagem sérica de sódio, potássio, cálcio e creatinina dentro da normalidade. Pacientes com disfunção hepática ou renal e aqueles que utilizavam fármacos que pudessem interferir com o bloqueio neuromuscular foram excluídos $^{8}$. Os pacientes foram divididos aleatoriamente em grupo pancurônio ou atracúrio dependendo do bloqueador neuromuscular que recebiam para complementar o bloqueio neuromuscular, inicialmente obtido com o pancurônio.

Durante a anestesia, os pacientes foram monitorizados com pressão arterial não invasiva, eletrocardiografia, oximetria de pulso e multianálise de gases. Todos receberam $1 \mathrm{mg}$ de midazolam por via venosa, logo após venóclise. Aindução da anestesia foi feita com 2 a $4 \mathrm{mg} \cdot \mathrm{kg}^{-1}$ de propofol e $5 \mu \mathrm{g} \cdot \mathrm{kg}^{-1} \mathrm{de}$ fentanil. Após a perda da consciência, os pacientes foram ventilados, sob máscara, com oxigênio a $100 \%$ e isoflurano a $0,5 \%$ (fração expirada). A monitorização da função neuromuscular foi realizada por acelerometria do músculo adutor do polegar, utilizando-se a seqüência de quatro estímulos (SQE) com estimulação supramáxima do nervo ulnar. Após estabilização, todos os pacientes receberam $0,08 \mathrm{mg} \cdot \mathrm{kg}^{-1} \mathrm{de}$ pancurônio administrado em cinco segundos para induzir o bloqueio neuromuscular. A manutenção da anestesia foi feita com óxido nitroso $60 \%$ em oxigênio e isoflurano a $0,5 \%$ (fração expirada). Nas situações em que o aumento da pressão arterial era maior que $20 \%$ administravam-se $2 \mu \mathrm{g} \cdot \mathrm{kg}^{-1}$ de fentanil. A ventilação foi ajustada para manter a $\mathrm{P}_{\mathrm{ET}} \mathrm{CO}_{2}$ entre 30 e $35 \mathrm{mmHg}$. O aquecimento dos pacientes foi controlado pelo método de convecção, por meio de manta térmica no tórax e nos membros superiores, de modo a manter a temperatura central entre 36 e $37^{\circ} \mathrm{C}$ e a periférica maior que $32{ }^{\circ} \mathrm{C}^{8,9}$.

Após a recuperação de $25 \%$ de $T_{1}\left(T_{1}=25 \%\right)$ do bloqueio inicial induzido pelo pancurônio (duração inicial 25\% - DI25\%), foi administrada dose complementar de $0,025 \mathrm{mg} \cdot \mathrm{kg}^{-1} \mathrm{de}$ pancurônio ou de $0,20 \mathrm{mg} \cdot \mathrm{kg}^{-1}$ de atracúrio, nos pacientes do grupo pancurônio e atracúrio, respectivamente (Figura 1).

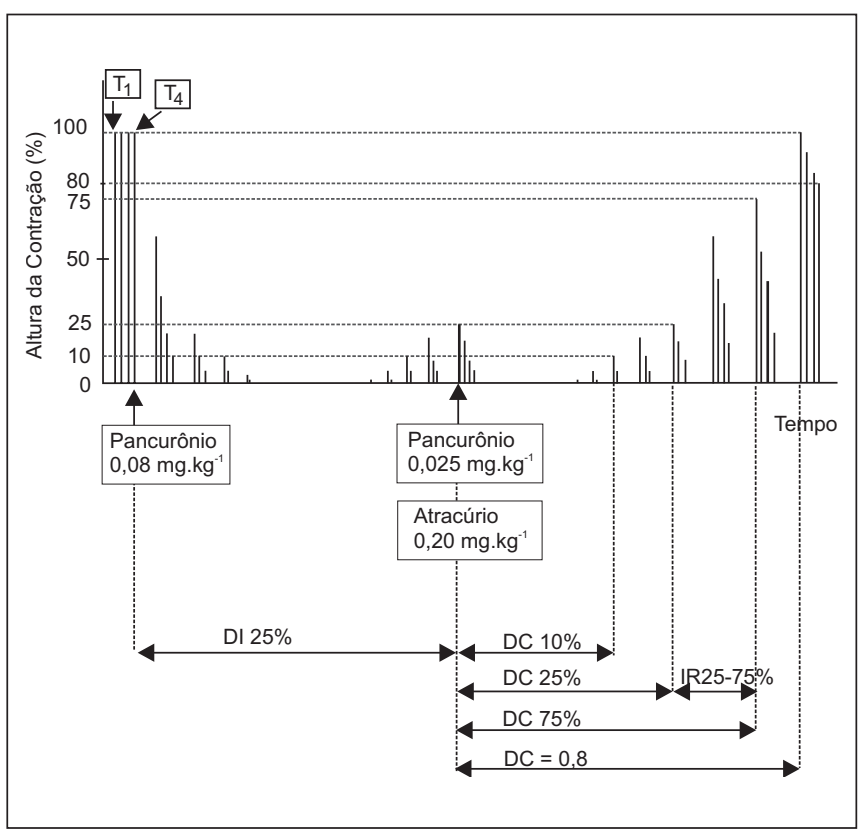

Figura 1 - Seqüência Experimental

O efeito da dose complementar de pancurônio ou de atracúrio na recuperação espontânea do bloqueio neuromuscular, foi avaliado através do tempo desde a administração da dose complementar até a recuperação da primeira contração à SQE igual a $10 \%, 25 \%, 75 \%$ e a relação entre a quarta e a primeira resposta da SQE $\left(T_{4} / T_{1}\right)$ igual a 0,8 . Esses tempos, após a dose complementar, foram denominados duração complementar (DC), conforme representado na figura 1.

Em todos os momentos em que foram anotados os dados referentes à monitorização da função neuromuscular foram também registrados os valores de pressão arterial, freqüência cardíaca, temperaturas periférica e central.

Os dados levantados foram submetidos ao teste Qui-quadrado para tabelas de contingência $2 X 2$ com o objetivo de comparar os grupos em relação à composição por gênero e estado físico (ASA). Teste $t$ de Student para comparar as médias dos grupos pancurônio e atracúrio em relação aos valores de idade, peso, altura, dosagem sérica dos íons sódio, potássio, cálcio e de creatinina.

Para análise da DI25\%, DC10\%, DC25\%, DC75\%, IR25-75\% e DC=0,8 foram aplicados a Análise de Variância de Friedman ${ }^{10}$ para comparar os deltas das doses compleVol. 54, No 3, Maio - Junho, 2004 
mentares, tanto para o grupo pancurônio como para o grupo atracúrio e o teste de Mann-Whitney ${ }^{10}$ para comparar os grupos pancurônio e atracúrio em todos os tempos estudados.

Fixou-se em 0,05 ou $5 \%$ o nível de rejeição da hipótese de nulidade.

\section{RESULTADOS}

Os grupos foram homogêneos quanto às características demográficas. No grupo atracúrio houve predomínio de pacientes ASA I (Tabela I).

\section{Tabela I - Dados Demográficos}

\begin{tabular}{lcc}
\hline & Grupo Pancurônio & Grupo Atracúrio \\
\hline Idade $(\mathrm{anos}){ }^{*}$ & $39,42 \pm 9,68$ & $41,00 \pm 13,18$ \\
Peso $(\mathrm{kg})^{*}$ & $68,52 \pm 12,60$ & $63,25 \pm 11,15$ \\
Altura $(\mathrm{m}){ }^{*}$ & $1,65 \pm 0,10$ & $1,62 \pm 0,08$ \\
Sexo & & 6 \\
$\quad$ Masculino & 7 & 10 \\
$\quad$ Feminino & 7 & $13^{* *}$ \\
Estado físico & & 3 \\
ASA I & 6 & \\
ASA II & 8 & \\
\hline
\end{tabular}

* Valores expressos em Média \pm DP

${ }^{* *} p<0,05$ (ASA I > ASA II, no grupo atracúrio)

A média dos tempos para a recuperação de $25 \%$ da primeira contração de $T_{1}$ (DI25\%) foi semelhante para os grupos pancurônio e atracúrio, 83,71 $\pm 28,15$ e 94,69 $\pm 35,91$, respectivamente.

As médias dos tempos para a recuperação de 10\%, 25\% e $75 \%$ após dose complementar de pancurônio ou de atracúrio foram semelhantes nos dois grupos. O índice de recuperação (IR25-75\%) foi maior no grupo pancurônio assim como a média dos tempos para a relação $T_{4} / T_{1}$ atingir $0,8(D C=0,8)$ (Tabela II).

Tabela II - Tempo (min) da Recuperação 10\%, 25\%, 75\% de $\mathrm{T}_{1}$ e de $\mathrm{T}_{4} / \mathrm{T}_{1}=0,8$ após Dose Complementar de Pancurônio ou Atracúrio (Média \pm DP)

\begin{tabular}{lcc}
\hline & $\begin{array}{c}\text { Grupo } \\
\text { Pancurônio }\end{array}$ & $\begin{array}{c}\text { Grupo } \\
\text { Atracúrio }\end{array}$ \\
\hline DC10\% & $45,00 \pm 15,50$ & $49,69 \pm 9,41$ \\
DC25\% & $61,64 \pm 18,58$ & $64,25 \pm 12,51$ \\
DC75\% & $94,00 \pm 28,52$ & $84,69 \pm 16,50$ \\
DC=0,8 & $176,86^{*} \pm 44,17$ & $141,50 \pm 29,57$ \\
IR25-75\% & $32,36^{*} \pm 13,76$ & $20,44 \pm 9,24$ \\
\hline
\end{tabular}

${ }^{*} p<0,05$

\section{DISCUSSÃo}

É sabido que os diversos anestésicos disponíveis sempre se mostraram inadequados para promover relaxamento muscular satisfatório, principalmente em procedimento intra-ab-

Revista Brasileira de Anestesiologia

Vol. 54, No 3, Maio - Junho, 2004 dominal, desde que administrados de modo seguro para o paciente. Este inconveniente foi contornado pelo emprego dos bloqueadores neuromusculares ${ }^{11}$.

$\mathrm{ADE}_{95}$ do pancurônio para bloqueio do músculo adutor do polegar durante anestesia com óxido nitroso é cerca de 0,06 $\mathrm{mg} \cdot \mathrm{kg}^{-112}$. Adose de intubação entre 0,08 e $0,12 \mathrm{mg}^{\mathrm{kg}}{ }^{-1}$ permite laringoscopia e intubação traqueal em 2 a 3 minutos. A duração clínica após dose de intubação de $0,08 \mathrm{mg} \cdot \mathrm{kg}^{-1}$ é de 86 minutos $^{13}$. Considera-se como principal desvantagem do pancurônio o fato de $85 \%$ de sua eliminação ser realizada pelos rins o que pode resultar em efeito prolongado nas situações de disfunção renal ${ }^{14}$.

O pancurônio, devido ao seu efeito prolongado, está associado com maior incidência de bloqueio neuromuscular residual na sala de recuperação pós-anestésica, quando comparado com bloqueadores de menor duração como o atracúrio ${ }^{15}$.

$\mathrm{O}$ atracúrio possui $\mathrm{DE}_{95}$ com valores entre 0,17 e 0,33 $\mathrm{mg} \cdot \mathrm{kg}^{-1} 16-19$. A dose de intubação é de 0,4 a $0,5 \mathrm{mg} \cdot \mathrm{kg}^{-13}$, e com esta dose a duração clínica é de 40 a 45 minutos ${ }^{18}$. Uma das vantagens do atracúrio em relação ao pancurônio é a menor duração clínica, o que promove retorno mais rápido da força motora, sendo portanto indicado quando há necessidade de relaxamento muscular menos prolongado ${ }^{15}$.

A pouca dependência de órgãos para sua eliminação e a menor duração clínica do atracúrio, comparativamente ao pancurônio, responde pela menor freqüência de bloqueio residual no pós-operatório; cerca de 4,3\% e 36,2\%, respectivamente ${ }^{15}$.

O bloqueio neuromuscular residual no pós-operatório relaciona-se com morbidade e mortalidade pós-operatórias. A incidência de complicações pulmonares no pós-operatório, entre os pacientes que recebem pancurônio, é de $11 \%$, enquanto que naqueles que recebem atracúrio é de $3,1 \%{ }^{20}$. A prática de complementar um bloqueio neuromuscular de longa duração com um agente de duração mais curta é um assunto que tem gerado interesse desde a introdução da succinilcolina na prática clínica em 1951. Entretanto, a utilização a succinilcolina com este propósito resultava em antagonismo ou intensificação do bloqueio, dependendo da fase de recuperação em que era administrada ${ }^{21}$. Considerando as dificuldades da utilização da succinilcolina para complementação do bloqueio neuromuscular adespolarizante de longa duração, ao término do procedimento cirúrgico, aliadas à introdução do atracúrio e do vecurônio na prática clínica, foi proposto que esses novos agentes substituíssem a succinilcolina para essa finalidade ${ }^{6,7}$.

Neste estudo foi pesquisado o efeito da complementação com atracúrio na recuperação espontânea do bloqueio neuromuscular induzido pelo pancurônio, avaliando-se a fase de recuperação inicial $\left(T_{1}=10 \%, 25 \%\right.$ e $\left.75 \%\right)$ e a de recuperação final $\left(T_{4} T_{1}=0,8\right)$.

Durante a recuperação do bloqueio neuromuscular, a resposta muscular evidencia-se a partir da recuperação de 10\% de $T_{1}$, quando há apenas uma contração da SQE. Nesta fase, $90 \%$ dos receptores nicotínicos da placa motora ainda estão sob ação dos bloqueadores neuromusculares. Quando a re- 
cuperação de $\mathrm{T}_{1}$ alcança $25 \%$, duas a três respostas à SQE podem ser observadas e $80 \%$ dos receptores nicotínicos ainda estão sob ação dos bloqueadores neuromusculares. A ocupação dos receptores ainda é alta, cerca de $75 \%$, quando a recuperação de $T_{1}$ atinge $100 \%{ }^{22}$.

Assim, o período de recuperação de $\mathrm{T}_{1}$ até $75 \%$ representa a fase inicial da recuperação do bloqueio neuromuscular. Aduração clínica ou duração 25 é o tempo, em minutos, decorrido desde a administração do bloqueador neuromuscular até que a primeira resposta à sequência de quatro estímulos recupere $25 \%$ do valor controle ${ }^{8}$. O tempo transcorrido para a recuperação de $\mathrm{T}_{1}$ entre $25 \%$ e $75 \%$ do controle é definido como índice de recuperação (IR25-75\%). É uma medida que tem sido utilizada como indicativo do grau de acumulação dos bloqueadores neuromusculares ${ }^{23}$.

Os valores obtidos, na primeira fase da seqüência experimental deste estudo, mostram que o método foi adequado uma vez que a duração clínica encontrada para a dose inicial de pancurônio foi semelhante à descrita por outros estudos que utilizaram anestesia venosa total ${ }^{5,13}$.

Após complementação com atracúrio, observou-se que os tempos durante a recuperação espontânea inicial de $T_{1}$ até $75 \%$ foram iguais nos dois grupos, à semelhança do que é descrito na literatura ${ }^{5,24}$. O fato de não ter havido diferença na recuperação do bloqueio quando complementado com pancurônio ou atracúrio permite concluir que houve um efeito potencializador do atracúrio pela administração prévia do pancurônio. Uma explicação deste efeito é que na fase inicial de recuperação do bloqueio, quando é realizada a dose complementar com um bloqueador de menor duração, a maioria dos receptores ainda está ocupada pelo bloqueador neuromuscular de longa duração e assim o bloqueio exibe predominantemente as características do agente administrado no início. Esta ação sinérgica e não apenas aditiva que ocorreu com o pancurônio também pode ser atribuída ao fato de que os bloqueadores neuromusculares não atuam de forma idêntica nos receptores da placa motora; há diferenças farmacocinéticas entre esses fármacos e presença de receptores nicotínicos pré e pós-sinápticos, aos quais os bloqueadores neuromusculares apresentam afinidades diferentes, dependendo da classe química a que pertencem ${ }^{25,26}$. Teoricamente, o efeito concomitante de dois fármacos, de classes químicas diferentes, potencializaria o bloqueio neuromuscular. Os resultados desse estudo mostram que, quando analisados isoladamente, os tempos para $\mathrm{T}_{1}$ recuperar $25 \%$ (DC25\%) e 75\% (DC75\%) não registram diferenças estatisticamente significantes entre os grupos; contudo, o IR25-75\% apresentou-se estatisticamente menor para o grupo atracúrio. Este resultado pode ser atribuído ao fato de que, embora o tempo para $T_{1}$ recuperar $75 \%$ não tenha sido estatisticamente diferente entre os grupos, houve uma tendência de desigualdades para o grupo atracúrio apresentar menor vaIor $(84,69 \pm 16,50$ vs $94,00 \pm 28,52)$. Quando avaliado o IR $25-75 \%$, esta desigualdade de valores tornou-se significante estatisticamente.
O índice de recuperação do atracúrio após $2 \mathrm{DE}_{95}$ é de $13 \mathrm{mi}-$ nutos ${ }^{17}$. Na vigência de bloqueio neuromuscular induzido pelo atracúrio, doses complementares de $0,16 \mathrm{mg} \mathrm{kg}^{-1}$ resultam em duração clínica de 28,6 minutos sem promover alteração no índice de recuperação ${ }^{5}$. Neste estudo, embora o IR25-75\% tenha sido menor no grupo atracúrio, se for considerado seu valor absoluto observou-se que houve aumento de $50 \%$ do seu valor após $2 \mathrm{DE}_{95}$.

Os aumentos do índice de recuperação e da duração clínica aliados ao fato de não ter ocorrido diferença estatística entre os grupos, quanto à recuperação de $\mathrm{T}_{1}$ em 10\%, 25\% e 75\%, demonstram que houve um efeito potencializador do pancurônio sobre o bloqueio complementar do atracúrio.

Outros estudos demonstraram efeito potencializador mais intenso do pancurônio registrando valores de índice de recuperação, após dose complementar do atracúrio, de cerca do dobro daquele após complementação com pancurônio ${ }^{5,24}$.

A avaliação da recuperação final do bloqueio neuromuscular até a relação $T_{4} / T_{1}$ atingir 0,8 justifica-se, porque os estudos sobre a recuperação objetivando o controle ventilatório, a mecânica respiratória e a proteção das vias aéreas sugerem que a recuperação adequada não ocorre até que $T_{4} / T_{1}$ seja igual ou maior do que $0,8^{8}$.

Neste estudo, o tempo para a relação $T_{4} / T_{1}$ atingir 0,8 no grupo que recebeu atracúrio como segundo bloqueador neuromuscular foi estatisticamente menor do que o do grupo que recebeu pancurônio o que clinicamente significaria recuperação final mais rápida com a administração de atracúrio. Este resultado difere de outro estudo que não evidenciou diferença na recuperação final de $T_{4} / T_{1}$ em 0,7 entre os dois grupos ${ }^{5,24}$. Considerando que esses autores avaliaram a recuperação de $T_{4} / T_{1}$ em 0,7 , não se pode assegurar que a recuperação final até $T_{4} / T_{1}$ atingir 0,8 manteria a mesma tendência.

Estudos demonstraram que o tempo para a recuperação de $\mathrm{T}_{1}$ em $25 \%$ até $\mathrm{T}_{4} / \mathrm{T}_{1}$ em 0,75 após dose de $0,5 \mathrm{mg} \cdot \mathrm{kg}^{-1}$ de atracúrio é de 28,4 minutos. Quando o atracúrio é administrado após o pancurônio, o tempo para a recuperação entre $T_{1}=25 \%$ até $T_{4} / T_{1}=0,70$ é de 72,6 minutos ${ }^{5,27}$. Neste estudo, este intervalo, ampliado para $T_{4} / T_{1}$ em 0,80 , foi de 77,3 minutos. Embora o grupo do atracúrio tenha apresentado tempo de recuperação final $20 \%$ menor que o do pancurônio, quando comparados os valores absolutos, entre os estudos, estes dados nos permitem inferir que a administração prévia de pancurônio potencializou o atracúrio. Assim, embora a complementação com atracúrio tenha promovido diminuição da recuperação final do bloqueio induzido pelo pancurônio, as características do bloqueio complementar ficaram mais semelhantes às do bloqueio de longa duração.

Nas condições deste estudo, a análise dos resultados da presente pesquisa permite afirmar que o bloqueio complementar com atracúrio na vigência de recuperação parcial do bloqueio neuromuscular induzido por pancurônio não altera a recuperação espontânea inicial e diminui em $20 \%$ o tempo para $T_{4} / T_{1}$ atingir 0,8 . 


\section{Effect of Atracurium on Pancuronium- Induced Neuromuscular Block Recovery}

Luís Fernando Rodrigues Maria, M.D.; Maria Angela Tardelli, TSA, M.D.; Rita de Cássia Rodrigues, TSA, M.D.

\section{INTRODUCTION}

Neuromuscular blockers are beneficial in general anesthesia to promote relaxation needed for tracheal intubation as well as to help surgical field approach with no need for deep anesthesia ${ }^{1}$.

Pancuronium is a steroid non-depolarizing neuromuscular blocker characterized by long-lasting neuromuscular block and ideal for surgeries lasting more than 4 hours ${ }^{2,3}$.

Atracurium belongs to the benzylisoquinolinic class with shorter duration as compared to pancuronium, with the additional advantage of less dependence on organs for its excretion ${ }^{4}$.

At intra-abdominal procedure completion, the degree of muscle relaxation in the surgical area is very important for adequate wall closing. Very often, additional neuromuscular blocker doses are needed in this stage of anesthesia to intensify muscle relaxation ${ }^{5}$. This is particularly important when neuromuscular block is complemented with long-lasting agents. The repetition of non-depolarizing neuromuscular blockers such as pancuronium could, as a function of its long duration, imply longer time for total motor strength and normal ventilatory function recovery. At this point, atracurium, and not pancuronium, to complement pancuronium-induced neuromuscular block could, in theory, result in faster motor function recovery ${ }^{6,7}$.

This study aimed at evaluating during partial pancuronium-induced neuromuscular block recovery, the effect of additional atracurium dose on spontaneous neuromuscular block recovery, as compared to additional pancuronium dose.

\section{METHODS}

After the Ethics Committee approval, participated in this study 30 patients of both genders, aged 18 to 65 years, with up to $30 \%$ normal weight variation, physical status ASA I or II, with normal serum sodium, potassium, calcium and creatinine. Exclusion criteria were patients with liver or kidney dysfunction and those under drugs which could interfere with neuromuscular block ${ }^{8,9}$. Patients were randomly divided in pancuronium or atracurium group depending on the neuromuscular blocker received to complement initial pancuronium-induced neuromuscular block.

Patients were monitored with noninvasive blood pressure, ECG, pulse oximetry and gases multianalysis. All patients received $1 \mathrm{mg}$ intravenous midazolam after venoclysis. Anesthesia was induced with 2 to $4 \mathrm{mg} \cdot \mathrm{kg}^{-1}$ propofol and $5 \mu \mathrm{g} . \mathrm{kg}^{-1}$ fentanyl. After loss of consciousness, patients were ventilated under mask with $100 \%$ oxygen and $0.5 \%$ isoflurane (ex- pired fraction). Neuromuscular function was monitored with accelerometry of adductor pollicis muscle using TOF stimulation with supramaximal ulnar nerve stimulation. After stabilization, all patients received $0.08 \mathrm{mg} \cdot \mathrm{kg}^{-1}$ pancuronium in five seconds to induce neuromuscular block. Anesthesia was maintained with $60 \%$ nitrous oxide in oxygen and $0.5 \%$ isoflurane (expired fraction). Fentanyl $\left(2 \mu \mathrm{g} . \mathrm{kg}^{-1}\right)$ was administered whenever blood pressure rise above $20 \%$, of control. Ventilation was adjusted to maintain $\mathrm{P}_{\mathrm{ET}} \mathrm{CO}_{2}$ between 30 and $35 \mathrm{mmHg}$. Patients' temperature was controlled by the convection method through a thermal blanket on the chest and upper limbs, to maintain central temperature between 36 and $37^{\circ} \mathrm{C}$ and peripheral temperature above $32{ }^{\circ} \mathrm{C}{ }^{8,9}$.

After $25 \% T_{1}$ recovery $\left(T_{1}=25 \%\right)$ of the initial pancuronium-induced block (initial duration 25\% - ID25\%) additional $0.025 \mathrm{mg} \cdot \mathrm{kg}^{-1}$ pancuronium or $0.20 \mathrm{mg} \cdot \mathrm{kg}^{-1}$ atracurium was administered to pancuronium and atracurium group, respectively (Figure 1).

The effect of additional pancuronium or atracurium dose on spontaneous neuromuscular block recovery was evaluated through time elapsed from additional dose administration to first TOF contraction equal $10 \%, 25 \%, 75 \%$ and fourth to first TOF response ratio $\left(T_{4} / T_{1}\right)$ equal 0.8 . These times after additional dose were called complementary duration (CD), as represented in figure 1.

Blood pressure, heart rate, peripheral and central temperature were recorded, in addition to neuromuscular function monitoring data.

Data were submitted to Chi-square test for contingency $2 \times 2$ tables, aiming at comparing groups by gender and physical status (ASA). Student's $t$ test was used to compare mean pancuronium and atracurium groups age, weight, height, serum sodium, potassium, calcium and creatinine levels.

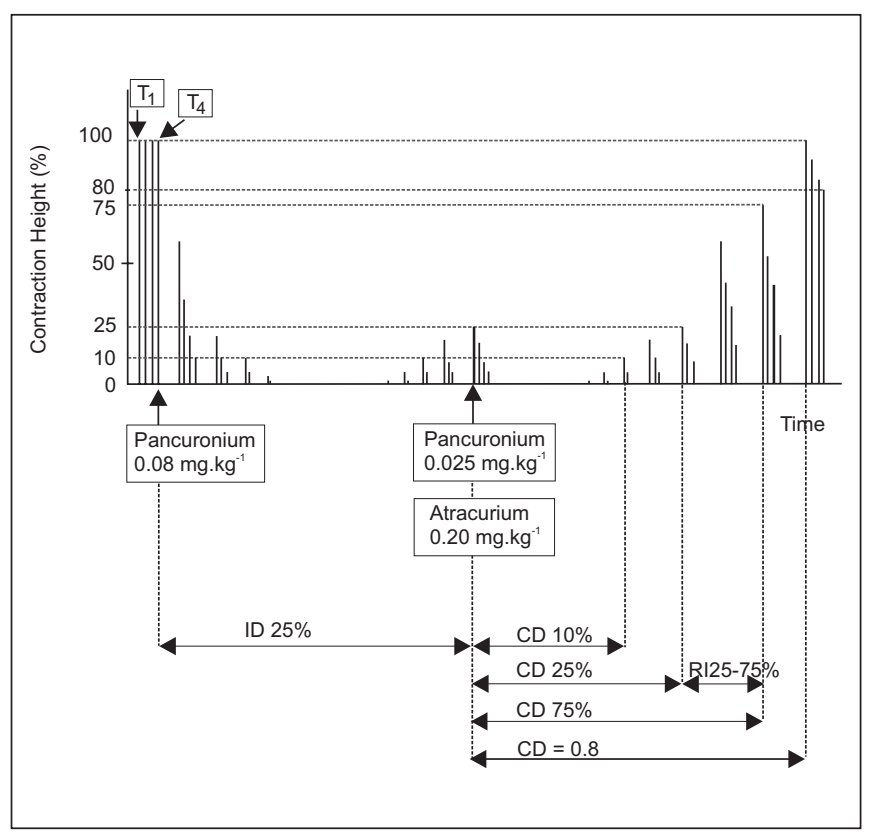

Figure 1 - Experimental Sequence 
Friedman's Analysis of Variance ${ }^{10}$ was used to analyze ID25\%, CD10\%, CD75\%, RI25-75\% and CD = 0.8, to compare additional doses deltas for both pancuronium and atracurium groups. Mann-Whitney ${ }^{10}$ test was used to compare pancuronium and atracurium groups in all studied moments, considering significant $p<0.05$ or $5 \%$.

\section{RESULTS}

Demographics data were homogeneous between groups. There has been predominance of ASA I patients in the atracurium group (Table I).

Table I - Demographics Data and Physical Status

\begin{tabular}{lcc}
\hline & Pancuronium Group & Atracurium Group \\
\hline Age (years) * & $39.42 \pm 9.68$ & $41.00 \pm 13.18$ \\
Weight $(\mathrm{kg}){ }^{*}$ & $68.52 \pm 12.60$ & $63.25 \pm 11.15$ \\
Height $(\mathrm{m}){ }^{*}$ & $1.65 \pm 0.10$ & $1.62 \pm 0.08$ \\
Gender & 7 & 6 \\
Male & 7 & 10 \\
Female & & $13^{* *}$ \\
Physical Status & 6 & 3 \\
ASA I & 8 & \\
ASA II & & \\
\hline
\end{tabular}

* Values expressed in Mean $\pm S D$

${ }^{* *} p<0.05$ (ASA I > ASA II, in atracurium group)

Mean time for $25 \%$ recovery of first $\mathrm{T}_{1}$ contraction (ID25\%) was similar for pancuronium and atracurium groups, $83.71 \pm$ 28.15 and $94.69 \pm 35.91$, respectively.

Mean time for $10 \%, 25 \%$ and $75 \%$ recovery after complementary pancuronium or atracurium dose was similar for both groups. Recovery index (RI25-75\%) was longer for pancuronium group, as well as mean time for $T_{4} / T_{1}$ to reach $0.8(C D=0.8)($ Table II).

Table II - Time (min) for 10\%, 25\%, 75\% $\mathrm{T}_{1}$ and $\mathrm{T}_{4} / \mathrm{T}_{1}=0.8$ Recovery after Complementary Pancuronium or Atracurium (Mean \pm SD)

\begin{tabular}{lcc}
\hline & Pancuronium Group & Atracurium Group \\
\hline CD10\% & $45.00 \pm 15.50$ & $49.69 \pm 9.41$ \\
CD25\% & $61.64 \pm 18.58$ & $64.25 \pm 12.51$ \\
CD75\% & $94.00 \pm 28.52$ & $84.69 \pm 16.50$ \\
CD 0.8 & $176.86^{*} \pm 44.17$ & $141.50 \pm 29.57$ \\
RI25-75\% & $32.36^{*} \pm 13.76$ & $20.44 \pm 9.24$ \\
\hline
\end{tabular}

* $p<0.05$

\section{DISCUSSION}

It is known that several available anesthetics when safely administered to patients are inadequate to promote satisfactory muscle relaxation, especially during intra-abdominal procedures. This inconvenient has been overcome by neuromuscular blockers ${ }^{11}$.
Pancuronium's $\mathrm{DE}_{95}$ for adductor pollicis muscle block during nitrous oxide anesthesia is approximately $0.06 \mathrm{mg}^{\mathrm{kg}} \mathrm{kg}^{-112}$. Intubation dose between 0.08 and $0.12 \mathrm{mg} \mathrm{kg}^{-1}$ allows for laryngoscopy in 2 to 3 minutes. Clinical duration after 0.08 $\mathrm{mg} \cdot \mathrm{kg}^{-1}$ is 86 minutes $^{13}$. Major pancuronium disadvantage is that $85 \%$ of its excretion is performed by kidneys, which may result in prolonged effect in the presence of kidney dysfunction ${ }^{14}$.

Due to its prolonged effect, pancuronium is related to higher incidence of residual neuromuscular block in post-anesthetic recovery units, as compared to shorter duration blockers such as atracurium ${ }^{15}$.

Atracurium's $\mathrm{DE}_{95}$ is 0.17 to $0.33 \mathrm{mg} \cdot \mathrm{kg}^{-1}{ }^{16-19}$. Intubation dose is 0.4 to $0.5 \mathrm{mg} \cdot \mathrm{kg}^{-13}$, and with this dose, clinical duration is 40 to 45 minutes $^{18}$. One advantage of atracurium over pancuronium is shorter clinical duration promoting faster motor strength recovery and being indicated when there is the need for less prolonged muscle relaxation ${ }^{15}$.

Atracurium's weak dependence on organs for excretion and shorter clinical duration, as compared to pancuronium, respond for the lower incidence of postoperative residual blockade; approximately $4.3 \%$ and $36.2 \%$, respectively ${ }^{15}$.

Postoperative residual neuromuscular block is related to postoperative morbidity and mortality. The incidence of postoperative pulmonary complications among patients receiving pancuronium is $11 \%$, while among those receiving atracurium it is $3.1 \%{ }^{20}$. The practice of complementing long-lasting neuromuscular blockers with shorter duration agents has gained interest since the introduction of succinylcholine in the clinical practice in 1951. However, succinylcholine for this purpose has resulted in blockade antagonism or intensification, depending on the recovery stage it was administered ${ }^{21}$. Considering the difficulties of using succinylcholine for complementation of long-lasting non-depolarizing neuromuscular blockers at surgery completion, and the introduction of atracurium and vecuronium in the clinical practice, it has been proposed that these new agents should replace succinylcholine for this objective ${ }^{6,7}$.

Our study has evaluated the effect of atracurium complementation in spontaneous pancuronium-induced neuromuscular block recovery, evaluating initial recovery $\left(\mathrm{T}_{1}\right.$ $=10 \%, 25 \%$ and $75 \%)$ and final recovery $\left(T_{4} / T_{1}=0.8\right)$.

During neuromuscular block recovery, muscle response is present as from $10 \%$ recovery of $T_{1}$, when there is only one TOF contraction. In this stage, $90 \%$ of end plate nicotinic receptors are still under the action of neuromuscular blockers. When $\mathrm{T}_{1}$ recovers to $25 \%$, two to three TOF responses may be observed and $80 \%$ of nicotinic receptors are still under the action of neuromuscular blockers. Receptors occupation is still high, approximately $75 \%$, with $\mathrm{T}_{1}$ recovery $=100 \%{ }^{22}$. So, $\mathrm{T}_{1}$ recovery to $75 \%$ is the initial neuromuscular blocker recovery stage. Clinical duration or duration 25 is the time in minutes elapsed from neuromuscular blocker administration to first TOF response to recover $25 \%$ of baseline value ${ }^{8}$. Time elapsed for $\mathrm{T}_{1}$ recovery between $25 \%$ and $75 \%$ of baseline is defined as recovery index (RI25-75\%). This measurement 
has been used to indicate the level of neuromuscular blockers build up ${ }^{23}$.

Values obtained in the first experimental sequence of this study have shown that the methodology was adequate since clinical duration for first pancuronium dose was similar to those described by other studies using total intravenous anesthesia $^{5-13}$.

After complementation with atracurium it has been observed that times during initial spontaneous $\mathrm{T}_{1}$ recovery to $75 \%$ were equal for both groups, in line with the literature ${ }^{5,24}$. The lack of difference in blockade recovery when complemented with pancuronium or atracurium allows the conclusion that there has been atracurium potentiating effect by previous pancuronium administration. One explanation would be that during initial blockade recovery stage, when dose is complemented with shorter duration blocker, most receptors are still occupied by the long duration neuromuscular blocker and blockade has predominantly the characteristics of the first agent. This synergistic and not only additive action with pancuronium may also be attributed to the fact that neuromuscular blockers do not act identically on end plate receptors; there are pharmacokinetic differences among these drugs and the presence of pre and post-synaptic nicotinic receptors, to which neuromuscular blockers have different affinities depending on their chemical class ${ }^{25,26}$. In theory, the concomitant effect of two drugs of different chemical classes would potentiate neuromuscular block.

Our results have shown that, when separately evaluated, times for $\mathrm{T}_{1}$ to recover 25\% (CD25\%) and $75 \%$ (CD75\%) were not significantly different between groups; however RI25-75\% was statistically lower for the atracurium group. This result could be attributed to the fact that, although time for $T_{1}$ to recover $75 \%$ has not been statistically different between groups, there has been a trend to inequalities for atracurium group to present lower value $(84.69 \pm 16.50$ vs. $94.00 \pm 28.52$ ). When RI25-75\% was evaluated, this inequality has become statistically significant.

Atracurium recovery index after $2 \mathrm{DE}_{95}$ is 13 minutes $^{17}$. In the presence of atracurium-induced neuromuscular block, additional $0.16 \mathrm{mg} . \mathrm{kg}^{-1}$ doses result in clinical duration of 28.6 minutes without recovery index changes ${ }^{5}$. In our study, although RI25-75\% has been lower in the atracurium group, if its absolute value is considered, $50 \%$ increase is observed after $2 \mathrm{DE}_{95}$.

Increased recovery index and clinical duration, together with no statistical difference between groups in $\mathrm{T}_{1} 10 \%, 25 \%$ and $75 \%$ recovery have shown a potentiating effect of pancuronium on complementary atracurium block.

Other studies have shown more intense potentiating effect of pancuronium with recovery indices after complementary atracurium doses of approximately twice the value found with complementary pancuronium ${ }^{5,24}$.

Final neuromuscular block recovery evaluation until $\mathrm{T}_{4} / \mathrm{T}_{1}$ equals 0.8 is justified because recovery studies aiming at ventilatory control, respiratory mechanics and airway protection suggest that there is no adequate recovery until $T_{4} / T_{1}$ is equal to or above $0.8^{8}$.
In our study, time for $T_{4} / T_{1}$ to reach 0.8 in the atracurium group has been statistically lower as compared to the pancuronium group, which would clinically mean faster final recovery with atracurium. This result is not in line with other studies which have not found differences in final $T_{4} / T_{1}$ recovery to 0.7 between groups ${ }^{5,24}$. Considering that these authors have evaluated $T_{4} / T_{1}$ recovery to 0.7 , we cannot assure that final recovery to 0.8 would maintain this same trend.

Studies have shown that time for $25 \% \mathrm{~T}_{1}$ recovery until $\mathrm{T}_{4} / \mathrm{T}_{1}=$ 0.75 after $0.5 \mathrm{mg} \cdot \mathrm{kg}^{-1}$ atracurium is 28.4 minutes. When atracurium is administered after pancuronium, recovery time from $T_{1}=25 \%$ to $T_{4} / T_{1}=0.75$ is 72.6 minutes $^{5,27}$. In our study, this interval enhanced for $\mathrm{T}_{4} / \mathrm{T}_{1}=0.80$ was 77.3 minutes. Although the atracurium group had final recovery time $20 \%$ shorter as compared to pancuronium, when absolute values are compared among studies, these data allow assuming that previous pancuronium administration has potentiated atracurium. So, although complementation with atracurium has promoted faster final recovery of pancuronium-induced neuromuscular block, complementary blockade characteristics were more similar to long duration blockade characteristics.

In the conditions of our study, analysis of results allows stating that complementary atracurium block during partial pancuronium-induced neuromuscular block recovery does not change initial spontaneous recovery and decreases in $20 \%$ time for $T_{4} / T_{1}$ to reach 0.8 .

\section{REFERÊNCIAS - REFERENCES}

01. Griffith HR, Johnson E - The use of curare in general anesthesia. Anesthesiology, 1942;3:418-420.

02. Baird WL, Reid AM - The neuromuscular blocking properties of a new steroid compound, pancuronium bromide. A pilot study. $\mathrm{Br}$ J Anaesth, 1967;39:775-780.

03. Savarese JJ, Miller RD, Lien CA et al - Pharmacology of Muscle Relaxants and their Antagonists, em: Miller RD - Anesthesia. $4^{\text {th }}$ Ed, New York, Churchill Livingstone, 1994;417-487.

04. Hunter JM, Jones RS, Utting JE - Use of atracurium in patients with no renal function. Br J Anaesth, 1982;54:1251-1258.

05. Nathan N, Bonada G, Feiss P - Potentiation of atracurium by pancuronium during propofol-fentanyl- $\mathrm{N}_{2} \mathrm{O}$ anesthesia. Acta Anaesthesiol Belg, 1996;47:187-193.

06. Aps C, Inglis MS - Peritoneal closure and atracurium. Anaesthesia, 1984;39:187.

07. Feldman SA - Peritoneal closure and atracurium. Anaesthesia, 1984;39:946.

08. Viby-Mogensen J, Engbaek J, Eriksson LI et al - Good clinical research practice (GCRP) in pharmacodynamic studies of neuromuscular blocking agents. Acta Anaesthesiol Scand, 1996;40:59-74.

09. Heier T, Caldwell JE, Sessler DI et al - The relationship between adductor policis twitch tension and core, skin, and muscle temperature during nitrous oxide-isoflurane anesthesia in humans. Anesthesiology, 1989;71:381-384.

10. Siegel S, Castellan Jr NJ - Non Parametric Statistics. $2^{\text {nd }}$ Ed, New York Mcgraw-Hill, 1988;399.

11. Duarte DF - Curarizantes - das suas origens aos dias de hoje. Rev Bras Anestesiol, 2000;50: 330-336. 
12. Engbaek J, Ording H, Pedersen T et al - Dose-response relationships and neuromuscular blocking effects of vecuronium pancuronium during ketamine anaesthesia; $\mathrm{Br} \mathrm{J}$ Anaesth. 1984;56:953-957.

13. Katz RL - Clinical neuromuscular pharmacology of pancuronium. Anesthesiology, 1971;34:550-556.

14. McLeod K, Watson MJ, Rawlins MD - Pharmacokinetics of pancuronium in patients with normal and impaired renal functions. Br J Anaesth, 1976;48:341-345.

15. Bevan DR, Smith CE, Donati F - Postoperative neuromuscular blockade: a comparison between atracurium, vecuronium, and pancuronium. Anesthesiology, 1988;69:272-276.

16. Payne JP, Hughes R - Evaluation of atracurium in anesthetized man. Br J Anaesth, 1981;53:45-54.

17. Basta SJ, Ali HH, Savarese JJ et al - Clinical pharmacology of atracurium besilate (BW33A): a new non-depolarizing muscle relaxant. Anesth Analg, 1982;61:723-729.

18. Katz RL, Stirt J, Murray AL et al - Neuromuscular effects of atracurium in man. Anesth Analg, 1982;61:730-734.

19. Gramstad L, Lilleaasen P - Dose-response relation of atracurium, ORG NC45 and pancuronium. Br J Anaesth, 1982;54: 647-651.

20. Pedersen T, Viby-Mogensen J, Ringsted C - Anaesthetic practice and postoperative pulmonary complications. Acta Anaesthesiol Scand, 1992;36:812-818.

21. Rouse JM, Bevan DR - Mixed neuromuscular block. A re-assessment using train-of-four stimulation. Anaesthesia, 1979;34:608-613.

22. Tardelli MA - Monitorização do Bloqueio Neuromuscular, em: Sociedade Brasileira de Anestesiologia. Curso de Educação à Distância em Anestesiologia. São Paulo, Office, 2002:177-190.

23. Bustamante Bozzo R - Recuperación Espontánea y Revision Farmacológica de los Relajantes Musculares, em: Alvarez Gomez JA, González Miranda F, Bustamante Bozzo R Relajantes Musculares. Madrid, Arám; 2000;129-137.

24. Whalley DG, Lewis B, Bedocs NM - Recovery of neuromuscular function after atracurium and pancuronium maintenance of pancuronium block. Can J Anaesth, 1994;41:31-35.

25. Waud BE, Waud DR, Phil D - Quantitative examination of the interaction of competitive neuromuscular blocking agents on the indirectly elicited muscle twitch. Anesthesiology, 1994;61: 420-427.

26. Amorós Arañó J, Solera Marin J, González Miranda F Interacciones, em: Alvarez Gomez JA, Gozález Miranda F, Bustamante Bozzo R - Relajantes Musculares en Anestesia y Terapia Intensiva. Madrid, Arán; 2000;165-186.
27. Erkola O, Karhunen U, Sandelin-Hellqvist E - Spontaneous recovery of residual neuromuscular blockade after atracurium or vecuronium during isoflurane anaesthesia. Acta Anaesthesiol Scand, 1989;33:290-294.

\section{RESUMEN}

Maria LFR, Tardelli MA, Rodrigues RC - Efecto de la Administración del Atracúrio sobre la Recuperación del Bloqueo Neuromuscular Inducido por el Pancuronio

JUSTIFICATIVA Y OBJETIVOS: Frecuentemente en cirugias abdominales, en la fase de cerramiento de la pared, hay necesidad de dosis adicionales de bloqueador neuromuscular. El objetivo de este estudio fue analizar, en la vigencia de recuperación parcial del bloqueo neuromuscular inducido por el pancuronio, el efecto de la administración de dosis complementares de atracúrio sobre la recuperación espontanea del bloqueo neuromuscular.

MÉTODO: Fueron estudiados 30 pacientes, divididos en dos grupos, 14 pacientes formaron el grupo pancuronio y 16 pacientes, el grupo atracúrio. La función neuromuscular fue monitorizada de forma continua por acelerometria del músculo aductor del pulgar, utilizando la secuencia de cuatro estímulos (SQE), a través de la estimulación supramáxima del nervio ulnar. La inducción de la anestesia fue hecha con propofol, fentanil, pancuronio 0,08 $\mathrm{mg}^{\mathrm{kg}} \mathrm{k}^{-1}$ y la manutención con $\mathrm{N}_{2} \mathrm{O}$ $60 \%$ en oxígeno e isoflurano en la concentración expirada de $0,5 \%$. Cuando la primera contracción de la secuencia de cuatro estímulos $\left(T_{1}\right)$ recuperó $25 \%$, el grupo pancuronio recibió pancuronio 0,025 mg. $\mathrm{kg}^{-1}$ y el grupo atracúrio, 0,20 mg. $\mathrm{kg}^{-1} \mathrm{de}$ atracúrio. Después de la dosis complementar fueron anotados los tiempos para recuperación espontanea de $T_{1}$ igual a $10 \%$, 25\%, 75\%, del índice de recuperación (IR25-75\%) y de la relación $T_{4} / T_{1}$ igual a 0,8 .

RESULTADOS: Los tiempos de recuperación espontanea después de la dosis complementar de pancuronio o atracúrio no divergieron cuando evaluados por la recuperación de $T_{1}$ en $10 \%(45,00 \pm 15,50$ vs 49,69 $\pm 9,41), 25 \%(61,64 \pm 18,58$ vs $64,25 \pm 12,51)$ y $75 \%(94,00 \pm 28,52$ vs $84,69 \pm 16,50)$. El IR25-75\% (32,36 $\pm 13,76$ vs $20,44 \pm 9,24)$ y el tiempo de recuperación de la relación $T_{4} / T_{1}=0,8(176,86 \pm 29,57 \mathrm{vs}$ $141,50 \pm 29,57)$ fueron mayores en el grupo en que la complementación fue realizada con pancuronio.

CONCLUSIONES: En las condiciones de este estudio, la complementación con atracúrio no promovió alteración en la recuperación espontanea inicial del bloqueo neuromuscular inducido por el pancuronio y promovió diminución de $20 \%$ en el tiempo de recuperación total. 